\title{
Ionization Injection and Acceleration of Electrons in a Plasma Wakefield Accelerator at FACET
}

\author{
N. Vafaei-Najafabadi, a) , C.E. Clayton ${ }^{1}$, K.A. Marsh ${ }^{1}$, W. An ${ }^{1}$, W. Lu ${ }^{1,2}$, W.B. \\ Mori $^{1}$, C. Joshi ${ }^{1}$, E. Adli ${ }^{3,4}$, J. Allen ${ }^{3}$, C.I. Clarke ${ }^{3}$, S. Corde ${ }^{3}$, J. Frederico $^{3}$, S. \\ Gessner $^{3}$, S.Z. Green ${ }^{3}$, M. Litos ${ }^{3}$, D. Walz ${ }^{3}$, M.J. Hogan ${ }^{3}$, V. Yakimenko ${ }^{3}$, P. \\ Muggli \\ ${ }^{1}$ University of California Los Angeles, Los Angeles, California, 90095, USA \\ ${ }^{2}$ Tsinghua University, Beijing, 100084, China \\ ${ }^{3}$ SLAC National Accelerator Laboratory, Menlo Park, California, 94025, USA \\ ${ }^{4}$ University of Oslo, 0316 Oslo, Norway \\ ${ }^{5}$ Max Planck Institute for Physics, 80805 Munich, Germany \\ ${ }^{a)}$ Corresponding author: navidvafa@ucla.edu
}

\begin{abstract}
Localized injection of electrons within a relativistic plasma wake can potentially produce an ultrashort, monoenergetic electron bunch. Recent experiments at the FACET facility explored the injection of helium electrons at the helium-lithium interface of a lithium heat pipe oven and the subsequent acceleration in the beam-produced plasma wake. Electrons accelerated to over $10 \mathrm{GeV}$ in $30 \mathrm{~cm}$ of plasma were observed as a distinct charge bunch.
\end{abstract}

\section{INTRODUCTION}

A high current electron beam can drive a plasma wakefield in the blowout regime, generating an accelerating gradient on the scale of tens of $\mathrm{GV} / \mathrm{m}$ [1]. A second electron bunch, placed at the right phase of this wake, can extract energy from the wakefield and be accelerated to high energies. Although there are a variety of methods proposed to create and inject this second electron bunch [2-5], they generally fall within two broad categories of external injection and ionization injection; self injection not being important for wakes produced by ultra-relativistic beams. External injection is also very difficult to realize in practice with ultra-relativistic beams. It requires generating a secondary bunch with sufficient energy outside the plasma, aligning it with the drive bunch, and placing it behind the drive bunch in an accelerating phase of the wake in plasma. In contrast, ionization injection of electrons produces charge within the wake, where they can quickly gain sufficient energy to be almost trapped by the wake. The requirement for ionization trapping is $\Psi_{f}<\Psi_{i}-m c^{2} / e$, where $\Psi=\Phi-c A_{z}$ is a pseudopotential with $\Psi_{i}$ and $\Psi_{f}$ referring to the value of this potential at the point of ionization (initial) and trapping (final), respectively, and $\Phi$ and $A_{z}$ are the electrostatic and electromagnetic potentials [6]. Additionally, if the location of injection is spatially confined to a small region, the resulting electron beams can have a monoenergetic spectra with a very small emittance, leading to a high brightness beam [2-4]. With these advantages, ionization injection has recently attracted great interest, and various methods of ionization within the wake have been examined [2-4]. In contrast to these methods, some of which require accompanying laser pulses that need to be aligned with the electron beam to an accuracy of few microns and temporally synchronized to tens of femtoseconds, here we present experimental observation of ionization injected electrons produced by taking advantage of the mismatch between the beam's and the wake's transverse characteristics. Using a simple experimental design, electrons accelerated to over $8 \mathrm{GeV}$ from a $30 \mathrm{~cm}$ plasma were observed with an emittance several times smaller 


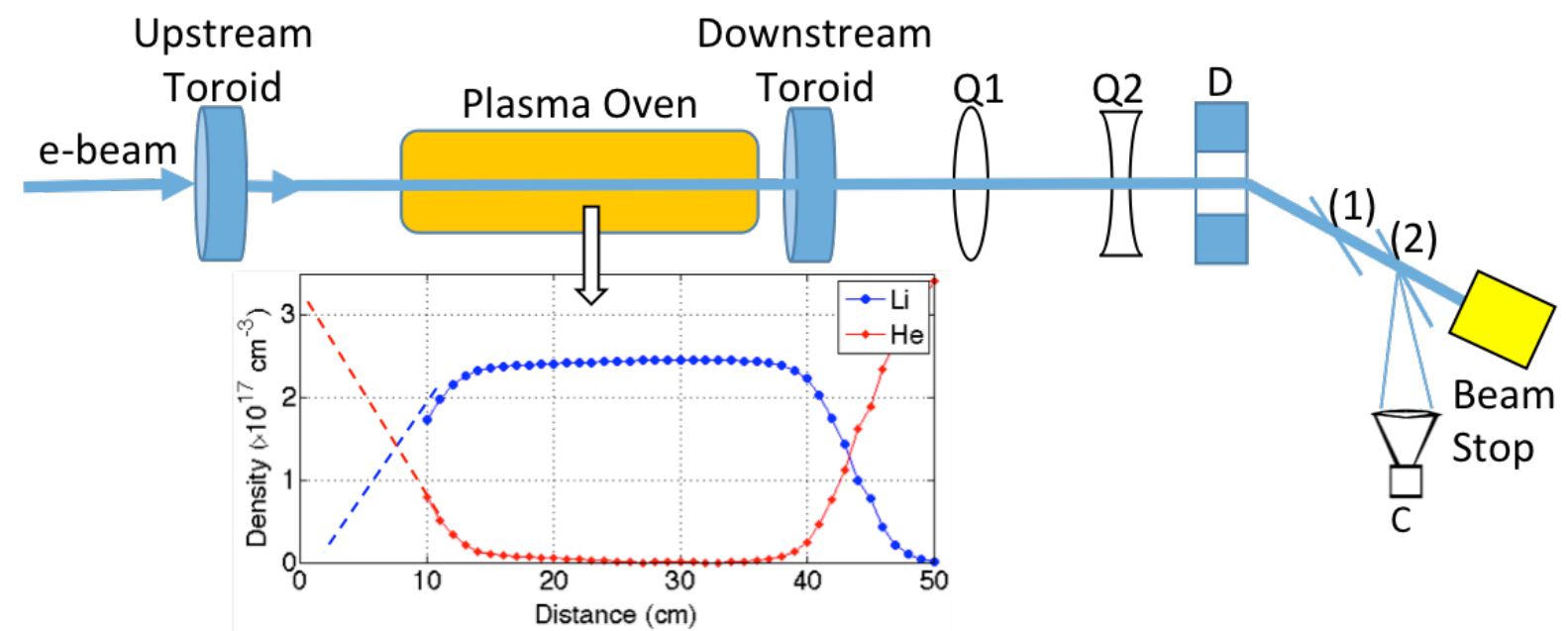

FIGURE 1. The schematic of the experiment. The electrons beam moves from the left to right. The upstream and downstream toroids measure the absolute charge before and after the plasma. The measured density profile of the neutral Li is shown accompanied with the estimated density of buffer gas (He). The imaging spectrometer consists of a dispersing dipole (D) and two imaging quadrupole magnets (Q1 and Q2). The Cherenkov light generated between two silicon wafers ((1) and (2)) is imaged into the $\mathrm{CCD}(\mathrm{C})$.

than that of the drive beam.

\section{EXPERIMENTAL SETUP AND DIAGNOSTICS}

The experiments were performed using the $20.35 \mathrm{GeV}$ electron beam produced at the FACET facility of the SLAC National Laboratory as the drive beam. The beam contained $2 \times 10^{10}$ electrons and an R.M.S size of $30 \times 30 \mathrm{x}$ $30 \mathrm{\mu m}^{3}$ at its waist. The plasma source consisted of a column of lithium (Li) vapor, which was produced in a heat pipe oven [7] operating at a peak temperature of $990^{\circ} \mathrm{C}$, corresponding to a peak Li density of $2.5 \times 10^{17} \mathrm{~cm}^{-3}[8]$. This hot vapor was confined by helium (He) gas, and the mixture produced a Li density profile with a $30 \mathrm{~cm}$ plateau at $2.5 \times 10^{17} \mathrm{~cm}^{-3}$ bounded by a ramp on either side. The ramp region contained both $\mathrm{Li}$ and $\mathrm{He}$, such that the combination of partial pressures added up to the pressure of cold background He, i.e. 32 Torr In these experiment. In other words, a decline in the Li density on either side of the plateau was accompanied by a rise in He density in the same region, as shown in the inset of Fig. 1. The location of the electron beam waist was set to the middle of the ramp.

The experimental diagnostic that was mainly used to observe the trapped electrons was the imaging spectrometer, shown schematically in Fig. 1. This spectrometer consisted of a dipole, which dispersed the electron beam in energy, and a pair of quadrupole magnets that enabled the imaging of various energies during the experiment. Along with the spectrometer, toroidal charge monitors were used upstream and downstream of the plasma, and the observation of difference between the two corroborated charge production during the interaction.

\section{INJECTED HELIUM ELECTRONS AS A SECONDARY BEAM}

The injected beam was identified on the energy spectrum as the beam that could be clearly distinguished from the drive beam. An example of such a beam is shown in Fig. 2, where an additional beam is clearly observed separated from the drive beam. The continuous feature from $20 \mathrm{GeV}$ to $9 \mathrm{GeV}$ is attributed to the drive beam, implying a peak energy loss of $11 \mathrm{GeV}$ for this beam. This feature is followed by a significant gap of about one $\mathrm{GeV}$, followed by a second electron beam. The significance of the gap is that it shows that the two beams are distinct and since this secondary beam is not part of the drive beam, it must have been generated during the interaction. This second bunch contains about $60 \mathrm{pC}$ of charge, and its centroid has an energy of $7.8 \mathrm{GeV}$ with the tail of the distribution reaching below $4.5 \mathrm{GeV}$. On the other hand, there is a distinct peak in the spectra with an rms energy spread of less than $0.5 \mathrm{GeV}$. Furthermore, the transverse size of the injected beam is very small, and separate analysis of emittance for similar beam indicates a normalized emittance smaller than $20 \mathrm{~mm}$-mrad [9], which is 


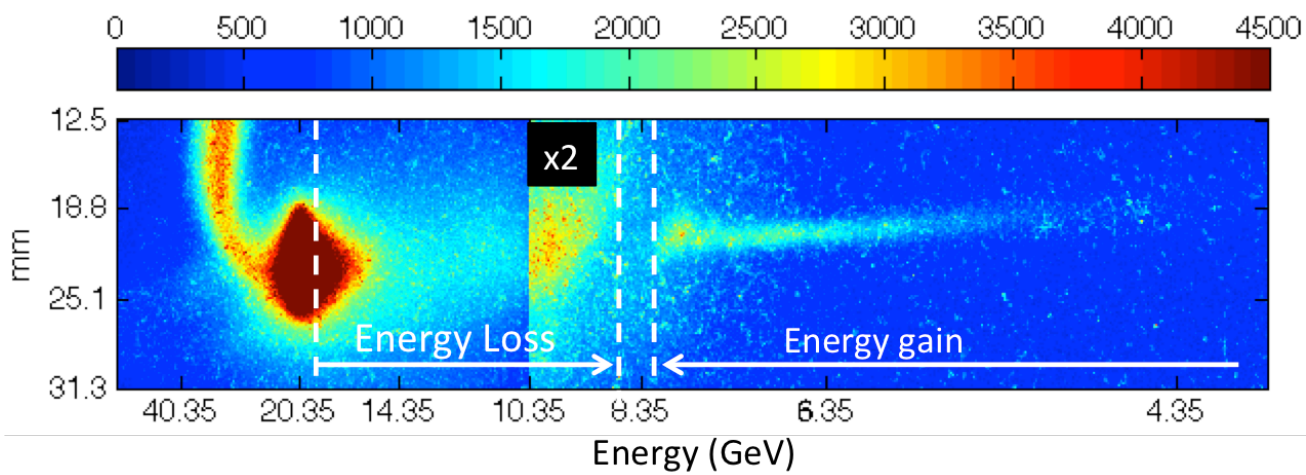

FIGURE 2. Experimental evidence of electron injection into the wake. The energy spectrum shows deceleration of the particles from the drive beam by about $9 \mathrm{GeV}$, a gap between 9 and $8 \mathrm{GeV}$, and a second beam with a peak at $7.8 \mathrm{GeV}$. The counts for electrons with energy less than $10.35 \mathrm{GeV}$ are multiplied by a factor of two to better show the two beams and the gap.

about five times smaller than that of the drive beam.

This secondary beam is thought to be generated via ionization injection due to pinching of the mismatched drive beam in the plasma. This is confirmed in OSIRIS PIC code simulations. The interaction starts when the electric field of the drive beam ionizes the Li vapor on the density ramp. As the drive beam propagates in this region, it forms a wake, which rapidly evolves into the blowout regime. The evolution of the R.M.S. transverse spot size of the beam in the resulting ion column can be calculated using

$$
\frac{d^{2} \sigma_{r}}{d z^{2}}+k_{\beta}^{2} \sigma_{r}=\frac{\varepsilon_{n}^{2}}{\gamma^{2} \sigma_{r}^{3}},
$$

where $\varepsilon_{n}$ is the normalized emittance, and $k_{\beta}$ is given by $\omega_{p} /(c \sqrt{2 \gamma})$. Because the beam in this experiment had a spot size larger than the matched spot size, given by $\sigma_{r}^{2}=\varepsilon_{n}\left(c / \omega_{p}\right) \sqrt{2 / \gamma}$, the beam radius oscillates. The transverse dynamic of a mismatched beam is well known and is explained in detail in other publications (see e.g. $[10,11])$. For parameters in our experiment, the beam radius pinches down to $\sigma_{r} \approx 2 \mu m$ on the up-ramp of $\mathrm{Li}$ plasma. Using the equation for the peak electric field for a bi-Gaussian pulse:

$$
E_{r, \max }=10.4\left[\frac{G V}{m}\right] \frac{N}{10^{10}} \frac{10}{\sigma_{r}[\mu m]} \frac{50}{\sigma_{z}[\mu m]},
$$

and taking $1.3 \times 10^{10}$ as the participating charge, which is consistent with the observations in the simulation (not shown) and previous experiments [12], the peak electric field can reach $75 \mathrm{GV} / \mathrm{m}$. Using the ADK ionization model [13] and integrating over the electron beam's temporal profile, the peak electric field required to ionize $10 \%$ of $\mathrm{He}$ and $\mathrm{Li}^{+}$are estimated at 65.2 and $290 \mathrm{GV} / \mathrm{m}$, respectively. Therefore, with the beam field in the experiment reaching $75 \mathrm{GV} / \mathrm{m}$, significant ionization of $\mathrm{He}$ can occur inside the wake. However, $\mathrm{Li}^{+}$cannot be further ionized, ruling out the ionization of $\mathrm{Li}^{+}$as a source of injected electrons. Simulations show that the electrons from the single ionized $\mathrm{Li}$ atoms are not injected into the ultra-relativistic wake because they do not obey the trapping condition ( $\left.\Psi_{f}<\Psi_{i}-m c^{2} / e\right)$. Once these electrons are ionized, they can be injected in the wake and are accelerated by the wake's high gradient.

\section{EFFECT OF PREIONIZATION}

Another method to show that the secondary bunch is comprised of ionization-injected electrons is to show that they disappear when the $\mathrm{He}$ is ionized before the arrival of the electron beam. When the He electrons are ionized before the formation of the wake, they, like the electrons from $\mathrm{Li}$, cannot inject into the wake. The high ionization potential of $\mathrm{He}^{+}$and $\mathrm{Li}^{+}$also preclude ionization and trapping of these electrons at the pinch point of betatron oscillation. In other words, the secondary beam will not form. FACET's $10 \mathrm{TW}$ class laser system [14] was therefore used during the experiment to preionize the plasma for every second electron pulse. The laser arrived $10 \mathrm{ps}$ earlier than the 


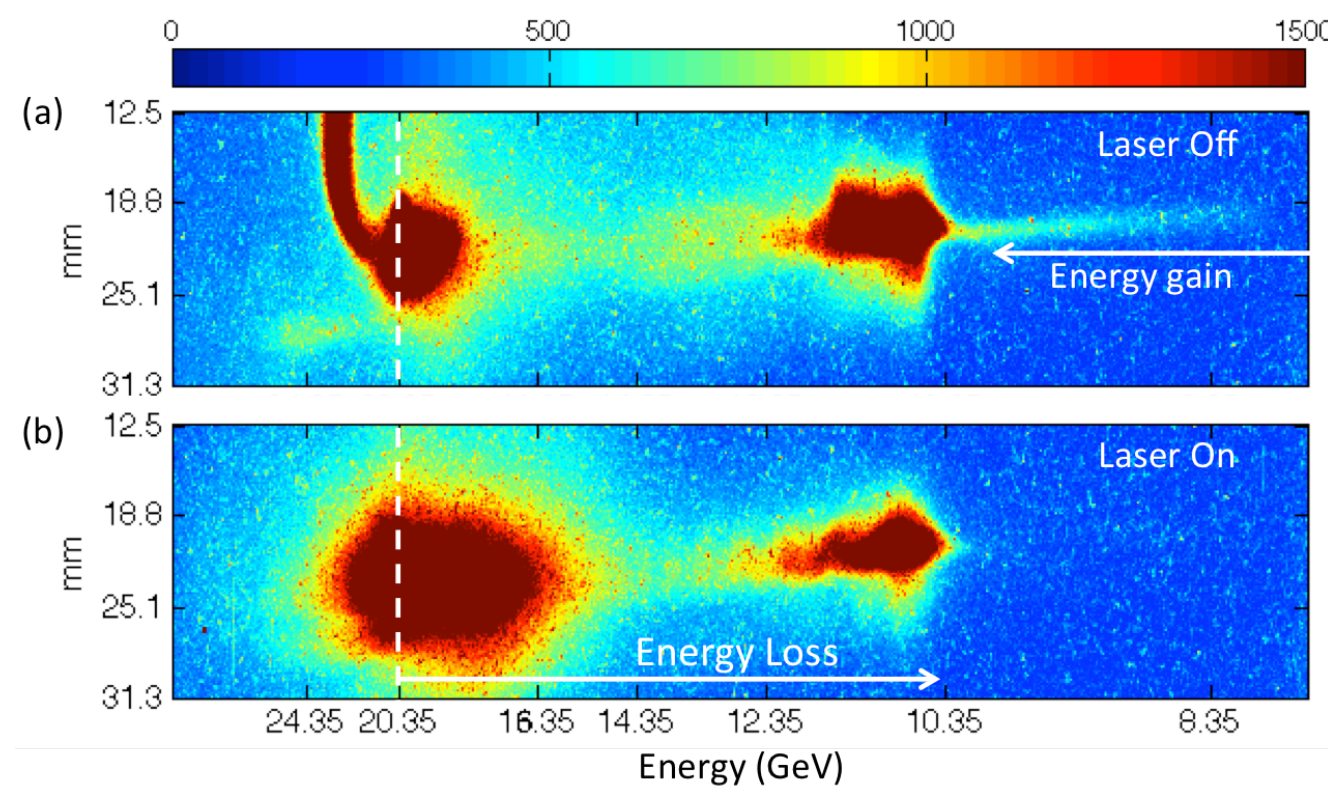

FIGURE 3. Effect of preionization on the experiment. (a) Laser off. The injected electrons have gained over $10 \mathrm{GeV}$ (b) Laser on. Only noise is observed at corresponding energies where electrons are observed on (a).

electron beam. The $500 \mathrm{~mJ}, 100 \mathrm{fs}$ laser pulse was focused using an axicon lens with 1.5 degree base angle, and was placed $1.2 \mathrm{~m}$ upstream of the beginning of the plateau region of plasma.

When the electron beam was preceded with the $10 \mathrm{TW}$ ionizing laser pulse, the beam of injected electrons disappeared over $80 \%$ of the time; indicating that the laser intensity was close to the ionization threshold of He. Examples of this phenomenon are shown in Fig. 3, where representative data samples from two successive datasets of 20 events (one with laser off and the other with laser on) are shown on the same color scale. Of the shots with laser off, the second bunch is observed in all the cases except for two; e.g. Fig. 3(a). In contrast, when the laser is on, the second bunch was absent from 18 of the 20 data events e.g. Fig. 3(b). It is noteworthy that the energy-loss feature of the drive beam in Fig. 3(b) is very similar to that observed in 3(a). This fact shows that the disappearance of the charge stripe depends directly on the presence of the laser and not just on factors such as the strength of the wake.

Let us therefore examine the interaction between the laser pulse and the He. The calculated laser intensity produced by the axicon at the top of the Li ramp reaches $2050 \mathrm{xI}_{0}$, where $\mathrm{I}_{0}$ is the laser intensity on the axicon. Since the laser beam at axicon has a flat-top spatial profile $40 \mathrm{~mm}$ wide, $\mathrm{I}_{0}=4.0 \times 10^{11} \mathrm{Wcm}^{-2}$. Therefore, the peak laser intensity, I, at the beginning of Li plateau, where the injection occurs, reaches $8.2 \times 10^{14} \mathrm{Wcm}^{-2}$. Using the laser pulse's envelope, the ionization rate obtained from the ADK model implies over $60 \%$ of the He is ionized, with the full ionization intensity estimated at $1.2 \times 10^{15} \mathrm{Wcm}^{-2}$. On the other hand, the resulting intensity due to the axicon lens is calculated for a laser pulse with uniform spatial profile. Real laser profiles are not smooth and it is therefore possible that shot to shot radial inhomogeneity and laser focusing in the plasma can lead to higher intensity and full ionization of He. The clear effect of the laser pulse on the interaction is circumstantial evidence for this possibility, while the fact that laser effectively removed trapped He electrons only $80 \%$ of the time indicates that the laser intensity is near the threshold for full ionization of $\mathrm{He}$.

\section{CONCLUSIONS}

Transverse dynamics of the FACET's beam were exploited to generate and inject electrons into a beam-driven plasma wakefield. Initial analysis shows that the emittance of the injected electron beam is several times smaller than the drive beam. To reduce the energy spread and improve the transformer ratio, it will be necessary to adjust the amount of the injected charge to optimize beam loading. This can be done by reducing the length of the injection site to be the same or slightly smaller than a single betatron wavelength. In other words, the length of injection region will need to be reduced from the current tens of centimeter to less than one centimeter. Beam loading can then be optimized by adjusting the amount of He gas, which is expected to improve both energy spread and the 
transformer ratio. Such solution will need a different plasma source than the current alkali vapor. If this is accomplished, it will be possible to generate a monoenergetic bunch with a much reduced emittance than the drive beam at tens of $\mathrm{pC}$ of charge using the same physical mechanisms as discussed in this paper.

\section{ACKNOWLEDGMENTS}

The work at UCLA was supported by DOE Grant No. DE-SC0010064. Work at SLAC was supported by the DOE Contract No. DE-AC02-76SF00515.

\section{REFERENCES}

1. I. Blumenfeld, et al., Nature 445, 741-744 (2007).

2. X. L. Xu, et al., Phys. Rev. Lett. 112, 35003 (2014).

3. B. Hidding, et al., Phys. Rev. Lett. 108, 35001 (2012).

4. J. Grebenyuk, et al., Nucl. Instrum. Meth. A. 740, 246-249 (2014).

5. M. J. Hogan, et al. New. J. Phys. 12, 055030 (2010).

6. A. Pak, et al. Phys. Rev. Lett. 104, 025003 (2010).

7. P. Muggli, et al., IEEE T. Plasma. Sci. 27, 791-799 (1999).

8. N. Vafaei-Najafabadi, et al., "Meter scale plasma source for plasma wakefield experiments," in Advanced Accelerator Concepts: $15^{\text {th }}$ advanced accelerator concepts workshop, AIP Conference Proceedings 1507, edited by R. Zgadzaj, E. Gaul and M. Downer (American Institute of Physics, Melville, NY, 2012), pp. 650655.

9. J. Frederico (private communication).

10. K. A. Marsh, et al., "Beam Matching to a Plasma Wake Field Accelerator using a Ramped Density Profile at the Plasma Boundary," in Particle Accelerator Conference 2005, Proceedings of the PAC 2005, edited by C. Horak. (IEEE, Piscataway, NJ, 2005) pp. 2702-2704.

11. C. E. Clayton, et al., Phys. Rev. Lett. 88, 154801 (2002).

12. N. Vafaei-Najafabadi, et al., "Suppression of the transformer ratio due to distributed injection of electrons in a plasma wakefield accelerator" in North American Particle Accelerator Conference (NA-PAC) 2013, Proceedings of PAC2013, c/o C. Petit-Jean-Genaz. (Joint Accelerator Conference Website (JACOW), Geneva, Switzerland, 2014), pp. 165-167.

13. D.L. Bruhwiler, Phys. Plasmas 10, 2022 (2003).

14. S. Z. Green, et al., Plasma Phys. Contr. F. 56, 084011 (2014). 